\title{
Increased Susceptibility to Postoperative PCA Morphine-Induced Respiratory Depression in a Patient with an Undiagnosed Traumatic Porencephalic Cyst - A Case Report
}

\author{
Cristina Petrișor ${ }^{1,2 *}$, Sebastian Trancă1,2, Andreea Cordoș²,3, Vasile Bințințan²,3 \\ 1 Anesthesia and Intensive Care II Department, "Iuliu Hațieganu" University of Medicine and Pharmacy Cluj-Napoca, \\ Romania \\ 2 Clinical Emergency County Hospital Cluj-Napoca, Romania \\ 3 Surgery Department I, "Iuliu Hațieganu" University of Medicine and Pharmacy Cluj-Napoca, Romania
}

\begin{abstract}
Introduction: Patient-controlled analgesia with morphine is routinely used for postoperative pain management. Due to the safety profiles of the technique, which are patient/disease related or technique/equipment related, severe respiratory depression requiring opioid antagonists or airway management are uncommon.

Case presentation: The case of a patient with right colon carcinoma who was operated on for hemicolectomy under general anaesthesia and who presented with apnoea, after postoperatively receiving an initial bolus of $1 \mathrm{mg}$ of morphine. A large post-traumatic porencephalic cyst of the left brain hemisphere, previously undiagnosed, was found on the computed tomography scan. We excluded human errors, technique and equipment factors, and the patient did not have any other predisposing conditions like sleep apnoea, obesity, recent head injury or concurrent use of other sedatives. Previously the patient had been entirely asymptomatic, and her increased susceptibility to respiratory depression was the only clinical manifestation of porencephaly.

Conclusion: Adult acquired porencephaly is seldom reported in the literature, clinical manifestations depending on the location and size of the cyst. In the present reported case, increased susceptibility to low-dose opioids might be associated with the structural and functional reorganisation of the brain after head trauma with the occurrence of the porencephalic cyst of the brain.
\end{abstract}

Keywords: patient-controlled analgesia, morphine, respiratory depression, porencephaly

Received: 26 March 2019 / Accepted: 26 April 2019

\section{INTRODUCTION}

Patient-controlled analgesia (PCA) with opioids, of which morphine is the most frequently administered drug, is routinely used for the management of postoperative pain. PCA implies the on-demand intermittent intravenous administration of opioids.[1] Due to the accepted safety of the technique, using morphine boluses of 1-2mg and lock-out intervals of 5-10 minutes, serious adverse events like respiratory depression are uncommon. PCA is commonly used to tailor opioid consumption according to the patient's needs and to lower opioid-associated side effects [2]. The reported incidences for overall depression are approximated at $0.1-0.8 \%[1,2]$, and this is even lower for severe respiratory depression.

The case of a patient with right colon carcinoma who was operated on for right-hemicolectomy under general anaesthesia and who presented apnoea, after receiving an initial bolus of $1 \mathrm{mg}$ of morphine is reported in this paper. A large porencephalic cyst of the brain, previously undiagnosed, was identified on a computed tomography (CT) scan. The associated structural and functional changes of the brain might be responsible for the increased susceptibility to opioid-induced respiratory depression. 


\section{CASE PRESENTATION}

A 62-years old female patient was admitted to hospital for a right colon adenocarcinoma with active bleeding and mild anaemia (haemoglobin $10 \mathrm{~g} / \mathrm{dL}$ ). During the pre-anaesthetic visit, the patient was conscious and oriented. No other pathologies were present at that time. From the history, it was established that she had suffered a head injury after a car crash twenty years previously following which she had a neurosurgical intervention for decompression. Neurological recovery was favourable, and she had no apparent neurologic deficits and no history of epilepsy, strokes, headache or psychiatric diseases. The patient had no medical documents which related to the traumatic event.

She was scheduled for surgery on the following day and refused to have an epidural catheter for analgesia. The proposed right hemicolectomy was carried out uneventfully under general anaesthesia with orotracheal intubation. After standard monitoring and pre-oxygenation, tracheal intubation was achieved in the sniffing position and anaesthesia induction accomplished intravenous with $0.2 \mathrm{mg}$ fentanyl (Fentanyl Torrex', Chiesi Pharmaceuticals GmbH, Austria), $200 \mathrm{mg}$ propofol (Propofol Fresenius', Fresenius Kabi Deutschland $\mathrm{GmbH}$, Germany) and $50 \mathrm{mg}$ rocuronium (Esmeron', MSD, Switzerland). The patient was mechanically ventilated in volume-controlled ventilation mode. Volatile maintenance of anaesthesia on sevoflurane was combined with intermittent boluses of fentanyl and rocuronium. The patient was hemodynamically, and respiratory stable during the threehours surgery and was transferred intubated and mechanically ventilated to the postanaesthesia care unit immediately after surgery. The patient was extubated one hour after arriving in the postoperative care unit, fully conscious and with adequate breathing.

The postoperative multimodal analgesic treatment included non-opiod analgetics and intravenous morphine (Morphine ${ }^{\circ}$ Zentiva, Czech Republic) on a patient-controlled analgesia (PCA) pump- Lifecare $4200^{\circ}$ (Abbott, USA). Morphine boluses were set at $1 \mathrm{mg}$, with a lock-out period of five minutes and a maximum amount of $10 \mathrm{mg}$ morphine per 4 hours. No background infusion of morphine was set. The patient complained of severe postoperative pain, graded 8 out of a maximum of 10 points and was instructed how to use the PCA device. After the initial 1mg morphine bolus, the bedside monitor signalled peripheral haemoglobin desaturation. The duty nurse, who noticed that the patient was in apnoea, and recorded that the peripheral haemoglobin saturation had dropped to $\mathrm{SpO} 230 \%$, blood pressure raised to $210 / 100 \mathrm{mmHg}$ which was accompanied by tachycardia (110 beats/minute) and miosis. Naloxone was not available for use at the time in the hospital. The patient was immediately intubated using propofol and esmeron rapid sequence induction, a pulsating structure was noticed in the left parietal head region. Blood pressure was invasively monitored and the patient received continuous propofol infusion and continuing mechanically ventilation.

The patient was referred for a cranial CT scan which revealed a large porencephalic cyst located in the frontoparietal and temporal left lobes, communicating with the left lateral ventricle (Figure 1A, B, C). The porencephalic cyst measured $7.8 \mathrm{~cm}$ in the sagittal plane and $5 \mathrm{~cm}$ in the coronal plane. The ventricular system presented asymmetry due to left lateral ventricle retraction. The frontoparietal-temporal craniectomy was obvious, with the corresponding bone defect.

The PCA pump was checked, and only the self-administered bolus of $1 \mathrm{mg}$ was missing, thus pump programming errors and pump failure were excluded.

The next day, the patient was extubated and subsequently received no further morphine. She was advised to have a neurological and neurosurgical consultation. The patient and the family disclosed that the patient had not had any prior cranial imaging since the initial traumatic event.

\section{DISCUSSION}

It is not entirely clear if the increased susceptibility to postoperative low-dose PCA morphine-induced respiratory depression in the patient with a large undiagnosed post-traumatic porencephalic cyst was due to functional changes in the pharmacokinetics and pharmacodynamics related to the structural changes of the brain.

A porencephalic cyst is a cavity in the cerebral hemisphere filled with cerebrospinal fluid that communicates directly with the ventricular system [3]. It is an extremely rare congenital condition reported in childhood and is thought to arise due to vascular occlusions during foetal development [4]. Even rarer, they can be diagnosed later in life or might be generated by other events during adulthood. Porencephalic cysts are uncommon intra-cranial cysts in adults and are seldom reported [5]. 

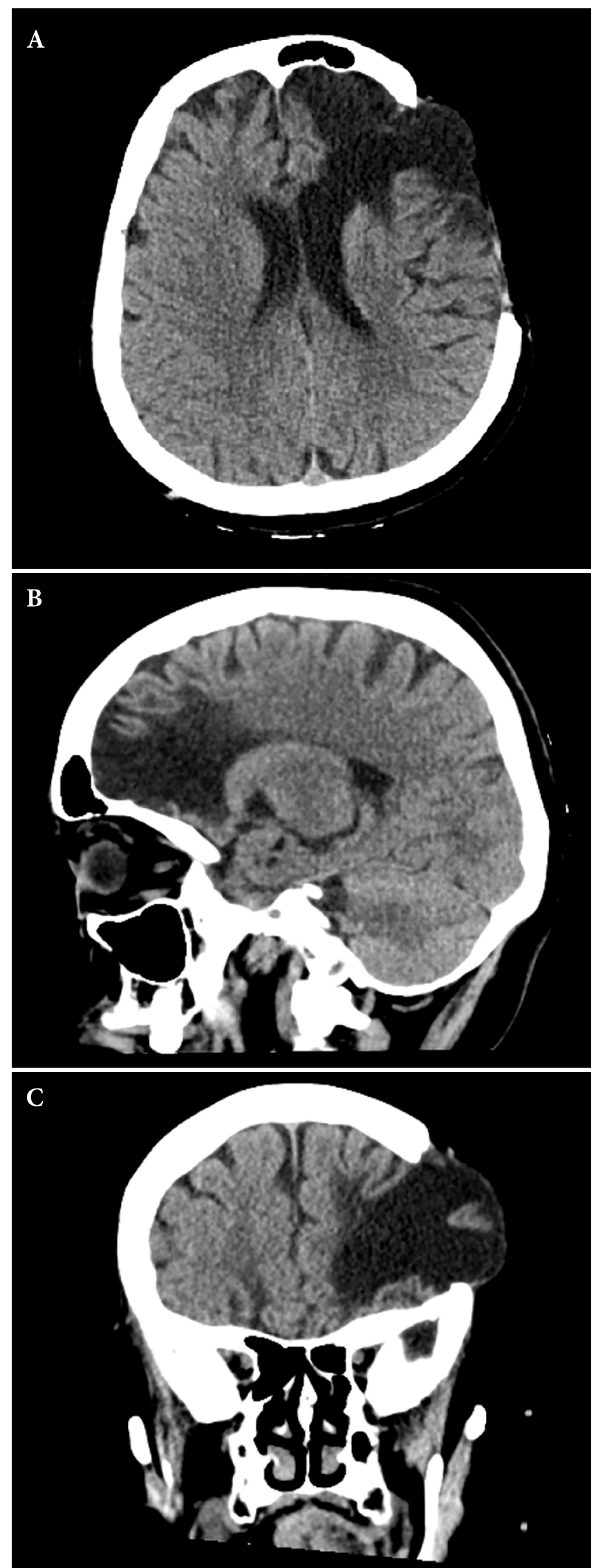

Fig. 1. CT scan of the brain in transverse (A), sagittal (B) and coronal (C) planes.
In adults, porencephalic cysts might be found accidentally after traumatic injuries of the brain, acquired porencephaly being usually caused by trauma, infarction, haemorrhage or focal encephalitis [6]. In these cases, the essential factor for the development of the lesion is focal destruction of the brain tissue that leads to encephalomalacia and cystic degeneration [6,7]. There are a some case reports in which porencephalic cysts were discovered after otitic meningitis, visual-field defects, strokes, seizures, and schizophrenia or other psychosis [3-5, 7-11]. Clinical features depend on the location of the cyst and its size while other patients may be entirely asymptomatic [5]. For an associated increase in intracranial pressure or intractable seizures, patients might require neurosurgical intervention [6].

Concerning the present case, brain tissue destruction was not apparent before the surgery. The patient had no neurological signs or history of neurologic complaints or headache. Neuroplasticity in adulthood has been previously reported in patients with left porencephalic cysts $[9,12]$. Similarly, the patient did not present with any neurological signs such as aphasia, alexia or ideational apraxia, which are typical for left hemisphere injuries. She had previously suffered head trauma during adulthood from which she recovered well. In patients with porencephaly, after brain tissue destruction and the reduction of the volume of the cerebral cortex, the contralateral side of the brain might take up the functions of the missing region [12]. These cases suggest that neuroplasticity can lead to functional adaptation of the brain even in adulthood and should be considered when brain recovery after trauma with functional reorganisation is discussed. In patients with porencephaly, the cerebral parenchyma around the cyst is stretched by the pulsations of the brain and the intracavitary pressure might be increased [6]. Pulsations simultaneously with apnoea, and the sympatheticallyinduced increase in blood pressure had been noted in the patient.

Though the patient's medical history specified no symptoms of increased intracranial pressure. It was considered appropriate advice for her to undergo neurological and neurosurgical evaluation afterwards.

There is evidence that PCA alone induces only little respiratory depression and the incidence of overall respiratory depression is $0.1-0.8 \%[1,2]$. Patients are prone to have mild desaturation, but they are less likely to progress to respiratory depression [13]. The incidences of severe respiratory depression requiring opioid an- 
tagonists and airway management is even lower. Deterioration of the breathing pattern in patients on PCA with opioids implies the stabilisation of the patient and sometimes, airway management. Another approach is to administer opioid antagonists [1], such as naloxone which unfortunately was not available in the hospital at the time of the incident.

The administration of on-demand opioid analgesia in the perioperative period is possible due to the safety settings of the PCA devices. Bolus doses are fixed, there is a lock-out period in which the drug can have a clinical effect and, if the patient becomes too sedated or drowsy, he will not be able to push the button [1]. Nevertheless, though the standard bolus dose of morphine was administered, respiratory depression manifested as apnoea and required airway management. Risk factors for severe respiratory depression after opioid PCA are patient/disease related or technique/equipment related [14-16]. Human errors, technique and equipment factors were considered and excluded. The patient had no other predisposing conditions like sleep apnoea, obesity, recent head injury or concurrent use of other sedatives. Also, these were absent after full recovery. The reversibility of the clinical situation and the return to normal physiologic variables, with absent clinical signs of neurological deterioration after recovery, demonstrate that apnoea was due solely to the initial morphine bolus.

Whether the patient's increased susceptibility had a direct correlation with the structural and functional changes of the brain tissue is not entirely clear, but all other causes have been ruled out. Apnoea due to a lowdose morphine bolus might be the first clinical manifestation of porencephaly in this case as the patient had no history of other neurological and psychiatric manifestations.

\section{CONCLUSION}

Increased susceptibility to low-dose opioids may possibly be associated with the structural and functional reorganisation of the brain after head trauma, resulting in with brain destruction and porencephalic cysts of the brain in adulthood. In the present reported case, increased susceptibility to opioid-induced respiratory depression was the only clinical manifestation of a large post-traumatic porencephalic cyst of the left brain hemisphere.
A review of the literature suggests that this is the first case report describing the association of porencephaly and increased susceptibility to apnoea induced by intravenous opioids. The reduction of brain tissue mass and related functional changes might represent predisposing factors for opioid respiratory depression. Patients with structural and functional alterations of the brain might need close respiratory monitoring in the postoperative period in order to detect abnormal breathing and prevent hypoxia.

\section{CONFLICT OF INTEREST}

None to declare.

\section{DEFERENCES}

1. Grass JA. Patient-controlled analgesia. Anesth Analg. 2005;101(5S):S44-61.

2. Stone M, Wheatley B. Patient-controlled analgesia. $\mathrm{Br} J$ Anaesth CEPD Reviews. 2002;2(3):79-82.

3. Yang DN, Townsend JC, Ilsen PF, Bright DC, Welton TH. Traumatic porencephalic cyst of the brain. Journal of the American Optometric Association. 1997;68(8):519-26.

4. Douzenis A, Rizos EN, Papadopoulou A, Papathanasiou M, Lykouras L. Porencephaly and psychosis: a case report and review of the literature. BMC Psychiatry. 2010;10(1):19.

5. Oommen AT, SeThy G, Minz NT, Patra J, Panda SS. Unusual presentation of porencephalic cyst in an adult. Journal of clinical and diagnostic research: J Clin Diagn Res. 2017;11(2): OD12.

6. Nakao N, Oiwa Y, Moriwaki H. Unusual Posttraumatic Porencephaly. Neurol Med-Chir. 1991;31(3):169-72.

7. Ryzenman JM, Rothholtz VS, Wiet RJ. Porencephalic cyst: a review of the literature and management of a rare cause of cerebrospinal fluid otorrhea. Otology and Neurotology. 2007;28(3):381-6.

8. Resende M, Abrunhosa J, Gonçalves P, Dos Santos JG, Moreira F, Dos Santos AG. Traumatic porencephalic cyst and cholesteatoma of the ear. J Laryngol Otol. 2000;114(11):8646.

9. de Oliveira-Souza R, Monteiro M, Pacheco P, et al. Right hemisphere dominance for language in a woman with schizophrenia and a porencephalic cyst of the left hemisphere. Neurocase. 2016;22(3):324-31.

10. Noyan OC, Şalçini C, Talu BS, Eryilmaz G. Porencephalic cyst and late-onset brief psychotic disorder. Case Reports. 2016; bcr2016215098.

11. Qureshi A, Jehangir A, York EP. Porencephalic cyst: a rare cause of new-onset seizure in an adult. J Community Hosp Intern Med Perspec. 2018;8(2):92-3. 
70 - The Journal of Critical Care Medicine 2019;5(2)

12. Danckert J, Mirsattari SM, Danckert S, et al. Spared somatomotor and cognitive functions in a patient with a large porencephalic cyst revealed by fMRI. Neuropsychologia. 2004;42(3):405-18.

13. Brose WG, Cohen SE. Oxyhemoglobin saturation following cesarean section in patients receiving epidural morphine, PCA, or meperidine analgesia. Anesthesiology. 1989;70(6):948-53.
Available online at: www.jccm.ro

14. Baxter AD. Respiratory depression with patient-controlled analgesia. Can J Anaesth. 1994;41(2):87-90.

15. Baird MB, Schug SA. Safety aspects of postoperative pain relief. Pain Digest. 1996;6(4):219-25.

16. Etches RC. Respiratory depression associated with patientcontrolled analgesia: a review of eight cases. Can J Anaesth. 1994;41(2):125. 